\title{
Funcionamiento familiar en relación con la conducta sexual de riesgo en adolescentes: Una revisión integradora de la literatura
}

\author{
Family functioning in relation to risky sexual behavior in adolescents: An \\ integrativereview of the literature
}

DOI: $10.46932 / \mathrm{sfjdv} 2 \mathrm{n} 2-208$

Received in: March 1st, 2021

Accepted in: May 30th, 2021

\author{
Alvaro Fabián Auquilla Guzmán \\ Psicólogo Clínico \\ Current Institution: Unidad de Posgrados Universidad Católica de Cuenca \\ Full address: Práctica Privada - Ecuador, Azuay, Cuenca, Yanucay \\ E-mail: alfast2009@hotmail.com
}

\begin{abstract}
RESUMEN
Introducción: La adolescencia es reconocida como una etapa donde suceden cambios acelerados a nivel biopsicosocial en el ser humano, durante esta etapa la interacción dentro del ámbito familiar permite que el adolescente aprenda distintas actitudes con respecto a cómo vivenciar la sexualidad, sin embargo, la búsqueda de nuevas experiencias puede favorecer la manifestación de conductas sexuales de riesgo poniendo en riesgo su salud sexual y reproductiva. Objetivo: Explorar las variables correspondientes a la funcionalidad familiar y su relación con las conductas sexuales de riesgo en población adolescente. Método: Se realizó una revisión integradora de la literatura en las bases de datos PubMed, Scopus, Web of Science, y Google Académico. Resultados: Los hallazgos encontrados pusieron en evidencia que las conductas sexuales de riesgo más representativas en los adolescentes fueron el embarazo adolescente, las infecciones de transmisión sexual, el número de parejas sexuales, el uso inconsistente de métodos anticonceptivos y el inicio temprano de relaciones sexuales, y que la disfuncionalidad familiar está caracterizada por un clima familiar hostil, bajos niveles de cohesión, muestras de afectividad escasas, estilos de crianza permisivo y autoritario, niveles bajos de monitorización y supervisión parental. Conclusión: A partir de la literatura revisada se destaca la importancia de potenciar los programas de educación sexual dirigidos a la población adolescente que incluyan las variables del funcionamiento familiar encontradas en esta investigación con la finalidad de potenciar los recursos con los que disponen los progenitores y fortalecer los vínculos filio-parentales.

Palabras clave: adolescencia, conducta sexual de riesgo, salud sexual, funcionamiento familiar, estilos de crianza
\end{abstract}

\begin{abstract}
Introduction: Adolescence is recognized as a stage where accelerated changes occur at the biopsychosocial level in the human being, during this stage the interaction within the family environment allows the adolescent to learn different attitudes regarding how to experience sexuality, however, the search for new experiences can favor the manifestation of risky sexual nbehaviors, putting their sexual and reproductive health at risk. Objective: The purpose of this integrative review of the literature was to explore the variables corresponding to family function and their relationship with risky sexual behaviors in the adolescent population. Method: An integrative review of the literature was carried out in the PubMed, Scopus, Web of Science and Google Scholar databases. Results: The findings found showed that the most representative sexual risk behaviors in adolescents were adolescent pregnancy, sexually transmitted infections, number of sexual partners, inconsistent use of contraceptive methods and early initiation of sexual intercourse, and that family dysfunction is characterized by a hostile family climate, low levels of
\end{abstract}


cohesion, few displays of affection, permissive and authoritarian parenting styles, low levels of parental monitoring and supervision. Conclusion: From the reviewed literature, the importance of promoting sexual education programs aimed at the adolescent population that includes the variables of family functioning found in this research is highlighted to enhance the resources available to parents and strengthen filio- parental ties.

Keywords: adolescence, risky sexual behavior, sexual health, family functioning, parenting styles

\section{INTRODUCCIÓN}

De acuerdo con la Organización Mundial de la Salud (2018), la adolescencia es una etapa de transición que abarca desde los 10 hasta los 19 años de edad, donde ocurren una serie de cambios tanto en la esfera física, psicológica como social. Esta etapa adquiere reconocimientoen el mundo occidental a partir del siglo XX, debido a que en construcciones sociales previas se consideraba que un infante llegaba a ser adulto cuando alcazaba una madurez física o cuandoaprendía una vocación (Papalia, Wendkos y Duskin, 2010).

En la actualidad y debido al proceso de globalización, la etapa de la adolescencia se ha tornado un fenómeno aceptado en la mayoría de sociedades, aunque esto no necesariamente implica que se la vivencie de igual manera, debido a que esta etapa se encuentra mediada fuertemente por factores culturales como la atribución de roles de género y el sexismo. Además, entran en juego de factores económicos como la pobreza y la desigualdad social queresultan importantes para alcanzar un desarrollo óptimo (Anderson, Dallal y Must, 2003; Papalia, Wendkos y Duskin, 2010).

En el ámbito de la sexualidad, factores como la aceptación corporal, la socialización homoy heterosocial y el establecimiento de apegos de carácter sexual suponen grandes retos para eladolescente. Además, a nivel cognitivo se evidencia un marcado egocentrismo e incluso una actitud de omnipotencia que a la vez se contrasta con sentimientos de vulnerabilidad por lo quela interacción de estos factores desemboca en la búsqueda constante de nuevas experiencias locual contribuye a la manifestación de conductas sexuales que impliquen un riesgo para su salud(Lefkowitz y Gillen, 2006; García, 2014).

Las conductas sexuales de riesgo son definidas como un conjunto de actitudes y prácticas que exponen a la persona a situaciones que conllevan potencial peligro para la salud propia y de terceros tales como infecciones de trasmisión sexual (ITS) o embarazos no planificados debido al uso inconsistente de métodos anticonceptivos (García, Menéndez, Fernández y Cuesta, 2012).

El Instituto Ecuatoriano de Estadísticas y Censos (INEC), reportó que en el Ecuador existenun total de 122301 madres adolescentes, donde el 34,1\% cuenta con instrucción primara mientras que el 56,7\% cuenta con instrucción secundaria, mientras que tan sólo en el año 2011se registró un total de 45708 partos en madres adolescentes, mientras que en 10 años el embarazo adolescente se incrementó en 2,3 puntos 
(2014). Esto supone un problema a nivel social y económico debido a que el embarazo puede conllevar a la interrupción en la formaciónacadémica limitando las oportunidades a futuro, además del estigma y la discriminación que pueden sufrir por parte de sus pares o incluso dentro del mismo ámbito familiar (Figueroa y Figueroa, 2017)

La presencia de conductas sexuales de riesgo en la adolescencia obedece a una serie de factores que interactúan entre sí. De acuerdo con Bronfenbrenner (1986), el adolescente es influenciado dentro de los ambientes más cercanos como su familia y el grupo de amigos, hastaaquellos contextos donde no participa de forma directa como los medios de comunicación y los marcos culturales e ideológicos. A este devenir se lo conoce como la teórica de ecológica cuyos niveles son denominados microsistema, mesosistema, exosistema y macrosistema.

De esta manera, la familia al ser uno de los ambientes primarios que conforman el microsistema, influye de forma importante en el desarrollo del adolescente (McCubbin y Thompson, 1996), sin embargo, un funcionamiento familiar inadecuado puede suponer un factor de riesgo en el desarrollo de la identidad del adolescente y favorecer la aparición de conductas sexuales de riesgo (Aquilino, 2006).

Entre las variables que se han estudiado para definir el funcionamiento familiar se encontróque la cohesión, la adaptabilidad y la comunicación son predictores fiables para evaluar este constructo (Olson, Sprenkle y Russell, 1979; Olson, Portner y Lavee, 1985). De este modo, familias que cuenten con un altos niveles de cohesión y adaptabilidad se ajustarán de mejor manera a las demandas del ciclo vital. Por otra parte, la falta de supervisión de los padres y la mayor autonomía otorgada a los jóvenes en familias monoparentales y mixtas pueden crear más oportunidades para la actividad sexual temprana (Feldman y Brown, 1993; Crockett, Raffaelli y Shen, 2006).

Por lo tanto, se considera pertinente analizar el funcionamiento familiar y cómo este influyeen el inicio o mantenimiento de conductas sexuales de riesgo como embarazos no planificadoso enfermedades de transmisión sexual, puesto que permitirá precisar conceptos sobre la temática además de conocer la situación actual de esta problemática.

\section{METODOLOGÍA}

Para esta investigación se realizó una revisión integradora de la literatura. De acuerdo con Whittemore y Knafl, este tipo de revisión de literatura permite la inclusión simultánea de investigaciones con diseño experimental y no experimental cuya finalidad recae en profundizarsobre un fenómeno de interés, lo cual permite obtener información fundamental para la comprensión del fenómeno de estudio (2005).

La búsqueda bibliográfica se realizó en las bases de datos electrónicas de PubMed, Scopus, Web of 
Science y Google Académico. Con el objetivo de realizar la búsqueda se empleó terminología en ingles debido a que es el idioma utilizado por las bases de datos, partiendo delas siguientes palabras clave: "family functioning", "risky sexual behaviour" "adolescence".

Los criterios de inclusión fueron i) publicaciones entre los años 2010 y 2020, ii) publicaciones en el idioma inglés y español, iii) publicaciones con muestra de población adolescente, iv) publicaciones pertenecientes al área de estudio de la psicología. Los criterios de exlusión fueron i) estudios con una muestra de adolescentes mayores a 19 años, ii) publicaciones a las que no se accedieron a la totalidad del texto, iii) estudios de intervención,

iv) tesis de pregrado y posgrado, capítulos de libros y entrevistas a profesionales.

Figura 1. Resultados de la búsqueda de literatura

Resultados de la búsqueda (número de visitas,
sin incluir duplicados):
PubMed $(\mathrm{n}=160)$,
Scopus $(\mathrm{n}=13)$,
Web of Science $(\mathrm{n}=17)$
Google Académico $(\mathrm{n}=103)$
Bola de nieve $(\mathrm{n}=5)$
$(\mathrm{N}=298)$

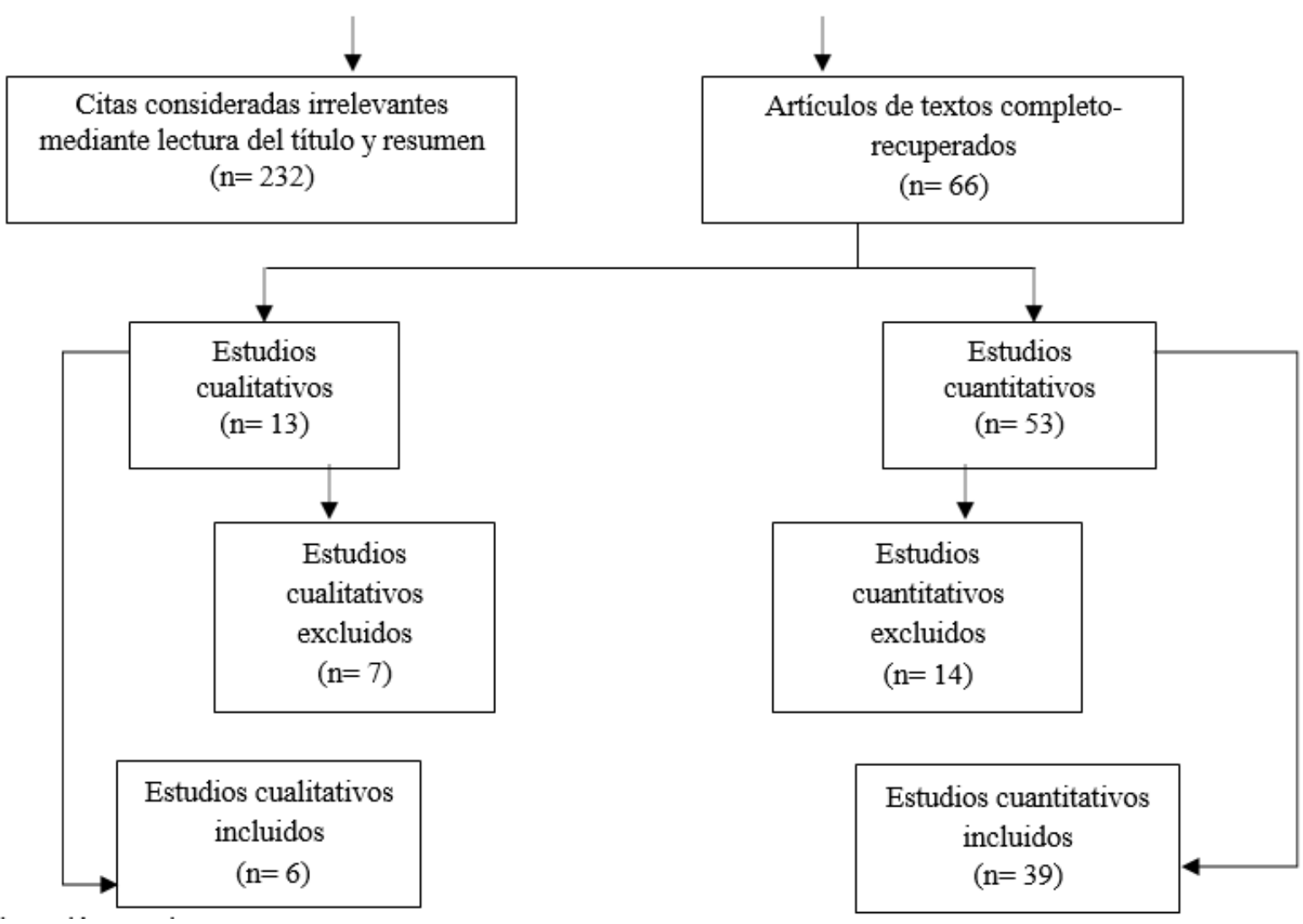

\section{RESULTADOS}

Con la finalidad de presentar los resultados, se agruparon los estudios alrededor de las principales 
Tabla 1

Resumen de los artículos seleccionados para esta revisión

\begin{tabular}{cc}
\hline $\begin{array}{c}\text { Autores y año } \\
\text { País }\end{array}$ & Diseño y Muestra \\
\hline Yi et al., 2010. Camboya & $\begin{array}{c}\text { Estudio cuantitativo } \\
\text { longitudinal. }\end{array}$ \\
& 1049 \\
García y Martínez, 2011. & Estudio de \\
comparativo, & transversal \\
& 77 familias gestantes \\
& 104 familias no \\
gestantes \\
González y de la Hoz, & Estudio \\
2011. Colombia & observacional de \\
& corte transversal \\
& 12302 \\
Hernández, Cargill y & Estudio de corte \\
transversal \\
observacional \\
descriptivo \\
\end{tabular}

Udell, Donenberg y

Emerson 2011

Estados Unidos

Instrumento
recolección

Resultado relevante

conductas sexuales de riesgo encontradas en cada una de las investigaciones. De esta manera 9 estudios están relacionados con el embarazo adolescente, 8 a infecciones de transmisión sexual, 5 al número de parejas sexuales, 13 al uso inconsistente de métodos anticonceptivos y 10 al inicio temprano de relaciones sexuales. Una descripción breve de los estudios encontrados con sus autores, el país procedente, diseño, muestra y herramientas de valoración se resumen de forma cronológica de la más antigua a la más reciente en la Tabla 1.

\section{Kalina et al., $2011 \quad$ Estudio comparativo Eslovaquia cuantitativo 2318}

\section{Murry, Chen, Gibbons y Gerrad, 2011. \\ Estados Unidos}
González et al., 2012. Colombia

Orcasita et al., 2012 Colombia

Ensayo de prevención aleatorizado y agrupado

150 familias control

172 en intervención Estudio observacional de corte transversal 7068

Estudio descriptivocorrelacional, de corte transversal
Estudio cuantitativo de tipo exploratorio 61 Family Support
Function Scale (FSFS)

The Assessment of Strategies in Families Effectiveness (ASF-E)

Adaptación del APGAR familiar

Prueba de percepción del funcionamiento familiar (FF-SIL)

Parenting Style Questionnaire (PSQ)

Encuesta diseñada para el estudio

Escala 36 de Apoyo 359 ítems revisada

Se utilizaron cuatro indicadores para evaluar la crianza comunicativa regulada

Adaptación del APGAR familiar

Adaptación del APGAR familiar
Una mayor probabilidad de comportamiento sexual de riesgo permaneció significativamente asociada con niveles más altos de haber presenciado violencia en la comunidad

Las adolescentes gestantes percibieron un nivel más alto de funcionalidad familiar en comparación adolescentes no gestantes

La disfunción familiar se asocia a una mayor frecuencia de conductas de riesgo en los adolescentes de bajo estrato socioeconómico en Bogotá

El promedio de edad para el inicio de relaciones sexuales era alrededor de los 15 años.

Diferentes relaciones entre la crianza de los hijos y las conductas sexuales de riesgo relacionadas con el VIH para niños y niñas en libertad condicional.

Los encuestados que vivían en una familia intacta o que tenían un mayor nivel de apoyo social de la familia tenían más probabilidades de seguir siendo vírgenes.
Los padres pueden influir indirectamente en el comportamiento de riesgo de los jóvenes a través de sus actividades de seguimiento

La disfunción familiar percibida por los adolescentes estaba asociada a una comunicación deficiente, al castigo físico y al maltrato emocional.

Los adolescentes quienes ya habían iniciado su vida sexual refirieron una falta de apoyo de su familia a nivel emocional e instrumental. 
Pérez et al., 2012

Chile

Zambrano, Vera y Flórez, 2012

Colombia

Voisin et al., 2012

Estados Unidos

Mmbaga, Leonard y

Leyna, 2012.

Tanzania

Malcolm et al., 2012

Estados Unidos

Rhucharoenpornpanich et al., 2012

Tailandia

Murry et al., 2013

Estados Unidos

Farid et al., 2013

Malasia

Kalina et al., 2013

Eslovaquia

Lee et al., 2013

Estados Unidos

Calderón et al., 2014

Cuba

Van de Bongardt et al., 2014

Los Países Bajos

Dessie, Berhane y Worku, 2014

Etiopía
Estudio de corte transversal

Estudio con enfoque cuantitativo, de tipo descriptivo

correlacional y

probabilístico

150 gestantes

Estudio cuantitativo correlacional 550

Estudio cuantitativo de tipo transversal 316

Estudio cuantitativo correlacional 171

\section{Estudio cuantitativo longitudinal 420 familias}

Estudio correlacional de tipo transversal 411 varones

Estudio transversal correlacional 1082

Estudio cuantitativo correlacional 1343

Revisión integradora de la literatura 17 artículos

Estudio de descriptivo observacional 58 gestantes

Estudio cuantitativo con un diseño longitudinal 900

Estudio transversal cuantitativo 641

\section{Ficha CLAP}

OPS/OMS

Escala de

Funcionalidad Familiar

(E-EFF)

Autoentrevista asistida por computadora

Cuestionario autoadministrado que se diseñó para el estudio

La escala de relaciones familiares.

La Escala de comunicación entre padres y adolescentes

Adolescent Health Study

Parenting Styles and Dimensions Questionnaire

Subescalas desarrolladas por Loeber, et al (1996) y (Simons et al, 1996)

Evaluación de Monitoreo de los Padres

Parental Bonding Instrument (PBI)

Artículos seleccionados de acuerdo con los criterios de inclusión y establecidos por los autores

Family adaptability and cohesion evaluation scales (FACES III)

Network of Relationship Inventory

Escala que evaluaba el seguimiento de los padres, los estilos de crianza y la comunicación
Los adolescentes que ya han comenzado la actividad sexual manifiestan una interacción deficiente entre los miembros de su familia, además de una precaria afectividad

En las adolescentes embarazadas se evidenció la presencia de familias disfuncionales, especialmente, la carencia de manifestaciones de cariño.

El monitoreo de los padres medió el inicio de la actividad sexual tanto para hombres como para mujeres adolescentes.

Los factores parentales que se asociaron significativamente con el debut sexual a una edad temprana es la comunicación

Los niveles más altos de funcionamiento familiar se asociaron directamente con niveles más altos de comunicación entre padres e hijos sobre el sexo

Los padres que practican más activamente sus creencias espirituales tienen menos probabilidades de hablar con sus adolescentes sobre temas sexuales

La falta de seguimiento y control de los padres puede ocasionar una disminución de las oportunidades para que los jóvenes estén expuestos a la socialización en los valores convencionales.

Las adolescentes mujeres con alta conexión familiar tenían más probabilidades de participar en conductas sexuales de riesgo. El seguimiento de los padres se asoció más fuertemente con la conducta sexual de riesgo que el apoyo social de los padres.

Los jóvenes latinos veían a sus padres como inaccesibles, demasiado estrictos, fuera de contacto con la realidad

Entre los factores predisponentes para el embarazo adolescente se encontró el ser parte de una familia extensa además de la disfunción familiar.

La calidad de la relación con los padres predice menores probabilidades de iniciación sexual y menos intención de tener relaciones sexuales

El alto control de los padres disminuye la probabilidad de estas prácticas de riesgo 
Elkington et al., 2014

Estados Unidos

Lavielle et al., 2014

México

Lee, Cintron, y Kocher, 2014

Estados Unidos

\section{Blanco, Cedre y Guerra, 2015 \\ Venezuela}

Ryan, Roman y Okwany, 2015

Sudáfrica

Dittus et al., 2015

Estados Unidos

Do et al., 2015

Vietnam

Lee et al., 2015

Estados Unidos

Okigbo et al., 2015

Kenia

Mmari et al., 2016

Estados Unidos y

Sudáfrica

Maness y Buhi, 2016

Estados Unidos

Crandall et al., 2017

Estados Unidos
Estudio de tipo
cualitativo

20

Estudio transversal analítico cuantitativo correlacional 909

Revisión integradora de la literatura 18 artículos

Estudios de casos y controles

40 primíparas y 75 nuligestas

Revisión sistemática de la literatura 20 artículos

Metaanálisis 30 artículos

Estudio cualitativo de tipo narrativo 31 padres

Revisión integradora de la literatura 18 artículos

Estudio cuantitativo longitudinal 1927

Estudio comparativo mixto de tipo correlacional 952

Revisión de la literatura 22 artículos

Estudio de corte longitudinal 450
Entrevistas a profundidad

la Escala de

Satisfacción Familiar por Adjetivos

Artículos seleccionados de acuerdo con los criterios de inclusión y establecidos por los autores

\section{Adaptación del}

APGAR familiar

Artículos seleccionados de acuerdo con los criterios de inclusión y establecidos por los autores

Artículos seleccionados de acuerdo con los criterios de inclusión y establecidos por los autores

\section{Grupo focal y entrevistas a profundidad}

Artículos seleccionados de acuerdo con los criterios de inclusión y establecidos por los autores

Cuestionario diseñado para la investigación, explora el monitoreo parental, la comunicación filio parental y la disciplina Cuestionario elaborado para la investigación centrado en la estructura familiar, el apoyo familiar y la violencia

Artículos seleccionados de acuerdo con los criterios de inclusión y establecidos por los autores

La funcionalidad familiar se evaluó utilizando 5 elementos del Chronic Stress Scale y 2 elementos del Family transition Project
Las familias de jóvenes que informaron comportamientos sexuales de riesgo a menudo describían relaciones que se caracterizaban por la tensión y el desapego El inicio precoz de la vida sexual fue más frecuente en adolescentes con expresión disfuncional del afecto en sus familias y estructura familiar monoparental

Las relaciones familiares más estrechas y una mayor participación de los padres se asocian con una serie de prácticas sexuales de bajo riesgo.

Los factores relacionados al embarazo adolescentes son el pertenecer a una familia con disfuncionalidad severa

Un menor control se relaciona con el no uso de condones en la actividad sexual tanto para niños como para niñas

Niveles más altos de control de los padres se asociaron con una mayor probabilidad de que los adolescentes usaran anticonceptivos.

Los padres creían que los adolescentes deben ser vigilados y controlados para evitar que se conviertan en hu hong, especialmente las niñas.

La actividad sexual prematura de los adolescentes se ve como una vergüenza y una amenaza para la armonía familiar

El control de los padres, la disciplina y la comunicación no predijeron la transición a la primera relación sexual para los hombres y si para las mujeres

La experiencia sexual de las adolescentes fue afectada por factores del entorno familiar y fue mayor entre las que vivían en hogares biparentales.

Asociación positiva significativa entre padres divorciados o separados y el embarazo entre los jóvenes.

Un alto estrés económico familiar predice la participación en conductas sexuales de riesgo mediadas por la autorregulación de los adolescentes. 
Coakley et al., 2017

Estados Unidos

$\begin{array}{cc}\text { Giusto, Green y Puffer, } & \text { transversal } \\ 2017 & 79 \text { diadas de } \\ \text { Kenia } & \text { cuidadores }\end{array}$

Moral de la Rubia y Garza, 2017

México

Sámano et al., 2017

México

De Jager y Naudé, 2018

Sudáfrica

Grossman et al., 2018

Estados Unidos

Angarita et al., 2019

Colombia

Causey y High, 2019

Estados Unidos

Chima et al., 2020

Sudáfrica

Ellis et al., 2020

Estados Unidos

Orihuela et al., 2020

Estados Unidos
Revisión de la

literatura

23 artículos

\section{Estudio cuantitativo \\ 79 diadas de \\ uidadores}

Estudio de corte transversal, correlacional 385

Estudio cualitativo 29 gestantes

Estudio cuantitativo correlacional 194

Estudio cualitativo 22

Estudio de tipo cualitativo mediante historias de vida 5

Estudio cuantitativo correlacional 1102 mujeres

Estudio cualitativo con un diseño exploratorio 13

Estudio cuantitativo longitudinal 1338

Estudio longitudinal prospectivo 4179
Artículos seleccionados de acuerdo con los

criterios de inclusión y establecidos por los autores

Una subescala de siete elementos del

Cuestionario para padres de Alabama Escala de comunicación entre padres y adolescentes

La Escala de Estilos Parentales e

Inconsistencia Parental Percibida (EPIPP)

\section{Entrevistas a} profundidad

Batería de encuestas adaptada compilada por Amoateng y Kalule (2013)

\section{Entrevista}

Entrevistas semiestructurada

Instrumentos de autoinforme centrado en la comunicación y el seguimiento de los padres

Entrevista

\section{Family Assesment}

Device (FAD)

Family adaptability and cohesion evaluation scales (FACES III)
El apoyo, el monitoreo y la comunicación de la familia se asociaron con un uso más constante del condón por parte de los jóvenes

A medida que aumentaba el respaldo de los padres a las creencias sexuales de riesgo, también aumentaba el respaldo de los hijos

La coerción física del padre y de la madre, este estaba asociado al mayor número de parejas sexuales.

La relación del adolescente con la familia fue de naturaleza diversa, caracterizadas por discusiones, discordias y poca comunicación real dentro del hogar

El monitoreo de los padres predijo un menor riesgo de representar un comportamiento sexual de riesgo

Los adolescentes eran más propensos a ver a la familia extendida que a los padres como personas fáciles de hablar

Las adolescentes embarazadas desarrollan su vida en familias con una estructura y una dinámica poco propicia

Asociación negativa entre el control de los padres y el riesgo sexual

Los factores familiares que influyen en las conductas sexuales de riesgo son la ausencia de progenitores, un entorno físico volátil y una mala comunicación

La depresión del cuidador y funcionamiento familiar adverso predijeron de forma única el desarrollo de conductas sexuales de riesgo

Los jóvenes que tenían un funcionamiento familiar más deficiente tenían más probabilidades de iniciar relaciones sexuales

Nota: Elaboración propia

\section{EMBARAZO ADOLESCENTE}

Los artículos revisados pusieron en evidencia que el embarazo adolescente es una de las principales conductas de riesgo en esta población. Los factores familiares asociados a este fenómeno son el pertenecer a familias altamente disfuncionales caracterizadas por escasas manifestaciones de cariño y poco tiempo compartido en familia donde la dinámica familiar mostraba niveles bajos de cohesión y sentimiento de pertenencia (Zambrano et al., 2012; Calderón et al., 2015). 
En cuanto al estilo parental se encontró que el estilo predominante es el autoritativo seguidodel permisivo, las adolescentes reportaron que sus madres se muestran más sobreprotectoras ytienen una mayor prohibición en comparación de los padres, sin embargo, se encontró que ambos progenitores ejercen coerción física como método de crianza siendo este un factor de riesgo (Moral de la Rubia y Garza, 2017; Angarita et al.,2019)

Además, en cuanto a la estructura y conformación del grupo familiar se encontró que aquellos hogares donde falta alguno de los progenitores presentan más riesgo de un embarazoadolescente, además de pertenecer a una familia con una madre o hermana con antecedentes de embarazo adolescentes, a esto se suma vivir en una condición de pobreza extrema y la pocaaccesibilidad a métodos anticonceptivos (González et al., 2012; Blanco et al., 2015; Maness yBuhi, 2016)

En contraste a los estudios anteriores, un clima familiar positivo también se encuentra ligadocon el embarazo adolescente (García y Martínez, 2011; Sámano et al., 2017) donde la adolescentes gestantes percibían el clima familiar como cohesionado y con mejores niveles decomunicación y compromisos, sin embargo, refirieron que luego del embarazo la comunicacióncon su pareja se vio deteriorada.

\section{INFECCIONES DE TRANSMISIÓN SEXUAL}

Por otra parte, los estudios centrados en las ITS como conducta de riesgo en la adolescencia encontraron que niveles bajos de monitorización familiar y un estilo de crianza de tipo permisivo caracterizado por niveles de comunicación deficiente y el desapego actúan como factor de riesgo, mientras que una mayor supervisión parental mostraba una menor incidencia de conductas sexuales de riesgo en los adolescentes (Murry et al., 2011; Udell et al.,2011; Mmbaga et al., 2012; Rhucharoenpornpanich et al., 2012; Elkington et al., 2014).

También se encontró similitudes en cuanto a la ubicación geográfica. Por ejemplo, en población afroamericana, Murry et al. 2013 destaca que los adolescentes reportaron en su entorno familiar un escaso apoyo e involucramiento por parte de sus progenitores, sin embargo, no se encontró una asociación significativa. Mientras que la población latina residente en Estados Unidos percibe a su familia como cercana proyectando sentimientos de lealtad, reciprocidad y solidaridad lo que a su vez se encuentra relacionado positivamente con el uso del condón. Estos resultados se muestran similares con la población afroamericana donde el monitoreo y la cohesión familiar fueron factores influyente en las prácticas sexuales saludablespara la prevención de ITS. (Lee et al., 2013; Lee et al., 2014).

\section{NÚMERO DE PAREJAS SEXUALES}

En lo que respecta al número de parejas sexuales, aquellos adolescentes que informaron unnivel 
más alto de autoestima positiva o apoyo social de amigos, pero un nivel más bajo de bienestar, apoyo social de la familia caracterizados por una expresión disfuncional de afecto, tenían más probabilidades de tener relaciones sexuales a una edad más temprana, además aquellas familias donde existe una monitorización baja sumando a una comunicación deficienteincrementaba la tasa de incidencia de tener múltiples parejas sexuales (Kalina et al., 2011; Dessie et al., 2014; Lavielle et al., 2014; Causey y High, 2019).

\section{USO DE MÉTODOS ANTICONCEPTIVOS}

El uso inconsistente de métodos anticonceptivos también fue un tema recurrente en las investigaciones revisadas, se evidenció que esta conducta estaba presente en familias disfuncionales marcadas por niveles bajos de cohesión, donde existía un menor apoyo tanto a nivel emocional como instrumental por parte de su familia (Orcasita et al., 2012; Dittus et al., 2015; Ellis et al., 2020; Orihuela et al., 2020). Sin embargo, este se encuentra mediado por otros factores como el nivel de monitoreo y control parental, además del apoyo instrumental yun alto nivel de conexión con sus pares (Coakley et al., 2017; Giusto et al., 2017; De Jager y Naudé, 2018).

Asimismo, estudios encontraron diferencias de género con respecto a esta variable, siendo las mujeres quienes reportaron un monitoreo parental más alto y una propensión más alta a participar en conductas sexuales de riesgo. Por otra parte, los hombres reportaron un mayor uso del preservativo de forma inconsistente (Voisin et al., 2012; Farid et al., 2013). Además, un menor control por parte del padre se relacionó significativamente con la primera relación sexual temprana entre las adolescentes y con la no utilización de condón durante la última relación sexual entre los varones (Kalina et al., 2013)

En contraste, otros estudios encontraron que no existe una asociación directa entre el uso demétodos anticonceptivos y el funcionamiento familiar, pero si con el uso de sustancias. (Yi et al., 2010; Malcolm et al., 2012).

\section{INICIO TEMPRANO DE RELACIONES SEXUALES}

La funcionalidad familiar también ha sido estudiada en relación con el inicio temprano de relaciones sexuales, las investigaciones alrededor de esta temática sugieren el pertenecer a hogares severamente disfuncionales está relacionado con un inicio más temprano, este tipo de familias se caracterizan por una escasa afectividad y niveles bajos de comunicación (González, y de la Hoz, 2011; Hernández et al., 2011; Pérez et al., 2012). Adicionalmente, se ha encontradoque una relación de alta calidad entre los adolescentes con su padres predice menores probabilidades de iniciación sexual y menos intención de tener relaciones sexuales (Van de Bongardt et al., 2014; Chima et al., 2020). 
Sobre las diferencias de género encontradas, los adolescentes varones que informaron una buena comunicación con sus madres tenían menor probabilidad de iniciar su vida sexual, en cuanto a las adolescentes mujeres, variables como el control parental, la disciplina y la comunicación con los padres predijeron la transición a la primera relación sexual (Okigbo et al., 2015). Otro hallazgo importante radica en que la comunicación de los padres indicó un retraso en la primera relación sexual, excepto cuando se recomendaron anticonceptivos en estasconversaciones, ya que se tradujo en la permisividad de los padres (Ryan et al., 2015; Grossmanet al., 2018)

Sobre las diferencias culturales, la revisión de la literatura en población asiático-americanareveló que dentro de estas familias se fomenta la dependencia mutua entre los miembros caracterizada por el apego natural y los comportamientos sexuales más conservadores, por lo que la actividad sexual prematura de los mismos es vista como una vergüenza, y una amenazapara la armonía familiar (Lee et al., 2015). Por otro lado, Mmari et al., 2016, encontraron que las adolescentes que fueron criadas por otros parientes tenían menos probabilidades de informar que habían tenido relaciones sexuales de forma prematura en comparación con las criadas por ambos padres biológicos.

\section{DISCUSIONES Y CONCLUSIONES}

El objetivo de esta revisión integradora de la literatura fue determinar la relación del funcionamiento familiar en las conductas sexuales de riesgo de los adolescentes. Los principales hallazgos muestran que el embarazo adolescente, el número de parejas sexuales, eluso inconsistente de métodos anticonceptivos y el inicio temprano de relaciones sexuales se posicionan como las conductas sexuales más frecuentes realizadas por los adolescentes.

En cuanto al embarazo adolescente, la literatura revisada sugiere que los factores familiares asociados son el pertenecer a familias altamente disfuncionales caracterizadas por niveles bajos de cohesión y de pertenencia, donde las muestras de afecto son limitadas, a esto se suman niveles deficientes de comunicación (González et al., 2012; Zambrano et al., 2012; Calderón et al., 2015). Los resultados entran en consonancia con lo referido por Olson, Sprenkle y Russell (1979), donde niveles bajos de cohesión y adaptabilidad familiar influyen de manera negativa en el comportamiento de los adolescentes, particularmente la noticia del embarazo esrecibida de forma negativa por parte de los familiares agravando aún más el clima familiar depor sí negativo.

Del mismo modo, en lo que respecta al número de parejas sexuales, factores como el apoyosocial de la familia, una estructura familiar monoparental, y el control parental deficiente se muestran como variables que incrementaban la probabilidad en los adolescentes (Kalina et al.,2013; Dessie et al., 2014; Causey y High, 2019). Los hallazgos encontrados dan pie a destacarla importancia de que los padres 
establezcan vías de comunicación centradas en la asertividady en la afectividad con la finalidad de crear un clima de confianza que permita un mejor controlde las actividades que realizan los adolescentes.

A esto se suman otras variables que hay que tomar en consideración como la condición socioeconómica, la calidad del vecindario, el estrés y depresión de los cuidadores, y la influencia de la familia extendida, que fueron abordadas en varias investigaciones revisadas (Mmari et al., 2016; Grossman et al. 2018; Ellis et al., 2020; Orihuela et al., 2020), demostrandotener un papel relevante en las conductas sexuales de riesgo realizadas por los adolescentes, pero que a su vez requieren de una mayor investigación con la finalidad de conocer de forma precisa el grado de influencia que ejercen.

Sobre las limitaciones encontradas en la investigación, se destaca que varios de los estudios revisados utilizan pruebas de medición no estandarizadas ni basadas en constructos teóricos específicos, optando por medir las variables del entorno familiar a través de cuestionarios diseñados específicamente para cada investigación, del mismo modo, otros estudios utilizabanlos instrumentos de forma incompleta evaluando aquellos ítems que se encontraban a la par con sus objetivos de investigación. Esto dificulta encontrar una definición clara de las variables correspondientes al funcionamiento familiar, lo que obstaculiza una comparación significativaentre los estudios que abordan estos conceptos.

La revisión muestra que la disfuncionalidad familiar caracterizada por niveles bajos de cohesión y de sentimientos de pertenencia, escasa afectividad y apoyo familiar, comunicacióndeficiente, estilos de crianza autoritativos y permisivos, niveles bajos de monitorización y supervisión parental, se presenta como un factor incidente en la práctica de conductas sexualesde riesgo en adolescentes.

Con base en lo anteriormente expuesto, se resalta la importancia de implementar y potenciarlos programas de educación sexual dirigidos a la población adolescente, abordándolos desde una perspectiva integradora e interdisciplinaria que incluya las variables del funcionamiento familiar encontradas en esta revisión, que además se complementen tomando en consideración la cultura y cosmovisión familiar. Intervenir en la funcionalidad familiar abordando sus múltiples componentes integrando además información verídica, libre de tabúes con respecto a la salud sexual y reproductiva, sería de gran beneficio puesto que dotará de herramientas y recursos a los padres para que puedan intervenir en esta etapa crucial en la que se encuentran sus hijos. 


\section{REFERENCIAS}

Anderson, E., Dallal, G., y Must, A. (2003). Relative Weight and Race Influence Average Age at Menarche: Results from Two Nationally Representative Surveys of US GirlsStudied 25 Years Apart. Pediatrics, 111. 844-850

Angarita, M., González, C., Cardona, H., Quitián, M., y Acero, E. (2019). Historias de vida familiar en madres adolescentes: estudio cualitativo realizado en el hospital Engativáese en Bogotá, Colombia. Revista Colombiana de Obstetricia y Ginecología, 70(1), 39-48

Aquilino, P. (2010). Adultez emergente y temprana. En D. Papalia, S. Wenkods, y R. Dusky, Desarrollo Humano. Mc Graw Hill.

Blanco, L., Cedro, O., y Guerra, M. (2015). Factores determinantes del embarazoadolescente. Odontol Pediatr, 14(2), 109-119

Bronfenbrenner, U. (1986). Ecology of the family as a context for human development: research perspectives. Developmental Psychology, 22(6), 723-742.

Calderón, I., Inalbes del Río, C., Rodríguez, O., Guisandes, A. (2014). Disfunción familiar engestantes adolescentes de un área de salud. MEDISAN, 18(11), 1523-1528

Causey, S., y High, B. (2019). An Exploration of the Ecological Context of Low-Income,Urban AfricanAmerican Adolescent Sexual Risk. Journal of Racial and Ethnic Health Disparities. http://dx.doi.org/10.1007/s40615-019-00633-5

Chima, F., Abayomi, H., Kwame, A., y Obisie, N. (2020) A qualitative assessment of the influence of family dynamics on adolescents' sexual risk behaviour in a migration-affected community, International Journal of Qualitative Studies on Health and Wellbeing, 15(1), 1-11. http://dx.doi.org/10.1080/17482631.2020.1717322

Coakley, T. M., Randolph, S., Shears, J., Beamon, E. R., Collins, P., y Sides, T. (2017).

Parent-youth communication to reduce at-risk sexual behavior: A systematic literature review. Journal of Human Behavior in the Social Environment, 27(6), 609- 624. http://dx.doi.org/10.1080/10911359.2017.1313149

Crockett, L., Raffaelli, M., y Shen, Y. (2006). Linking Self-Regulation and Risk Proneness toRisky Sexual Behavior: Pathways through Peer Pressure and Early Substance Use.

Journal of Research on Adolescence, 16(4), 503-525

De Jager, M., y Naudé, L. (2018). Antecedents of risk-taking behaviour among South Africanadolescents. Journal of Psychology in Africa, 28(5), 382-388. http://dx.doi.org/10.1080/14330237.2018.1523336

Dessie, Y., Berhane, Y., y Worku, A. (2014). High parental monitoring prevents adolescents from engaging in risky sexual practices in Harar, Ethiopia. Global Health Action, 7(1), 25724. http://dx.doi.org/10.3402/gha.v7.25724

Dittus, P. J., Michael, S. L., Becasen, J. S., Gloppen, K. M., McCarthy, K., y Guilamo- Ramos, V. (2015). Parental Monitoring and Its Associations With Adolescent SexualRisk Behavior: A Meta-analysis. PEDIATRICS, 136(6), e1587-e1599. http://dx.doi.org/10.1542/peds.2015-0305 
Elkington, K. S., Belmonte, K., Latack, J. A., Mellins, C. A., Wasserman, G. A., Donenberg, G. R., y Hirsch, J. S. (2014). An Exploration of Family and Juvenile Justice Systems to Reduce Youth HIV/STI Risk. Journal of Research on Adolescence, 25(4), 700-716.http://dx.doi.org/10.1111/jora.12163

Ellis, B. J., Shakiba, N., Adkins, D. E., y Lester, B. M. (2020). Early external-environmentaland internalhealth predictors of risky sexual and aggressive behavior in adolescence: An integrative approach. Developmental Psychobiology, 1-16. http://dx.doi.org/10.1002/dev.22029

Farid, N. D. N., Rus, S. C., Dahlui, M., Al-Sadat, N., y Aziz, N. A. (2014). Predictors of sexual risk behaviour among adolescents from welfare institutions in Malaysia: a cross sectional study. BMC Public Health, 14(3), 2-8. http://dx.doi.org/10.1186/1471-2458-14-s3-s9

Feldman, S., y Brown, N. (1993). Family influences on adolescent male sexuality: themediational role of selfrestraint. Social Development, 2(1). 15-35.

Figueroa, L., y Figueroa, L. (2017). Conductas sexuales de riesgo en adolescentes desde elcontexto cubano. Revista de Ciencias Médicas de Pinar del Río.

García, A., y Martínez, C. (2011). Efectividad de la funcionalidad familiar en familias con adolescentes gestantes y adolescentes no gestantes. Avances en enfermería, 19(1), 75-86

García, E., Menéndez, E., Fernández, P., y Cuesta, M., (2012). Sexualidad, Anticoncepción yConducta Sexual de Riesgo en Adolescentes. International Journal of Psychological Research, 5(1), 79-87

García, M. (2014). Las conductas sexuales de riesgo de los adolescentes españoles. RqREnfermería Comunitaria, 2(2), 38-61

Giusto, A. M., Green, E. P., y Puffer, E. S. (2017). Associations Between Fathers' and Sons'Sexual Risk in Rural Kenya: The Potential for Intergenerational Transmission.

Journal of Adolescent Health, 61(2), 219-225. http://dx.doi.org/10.1016/j.jadohealth.2017.02.007

González, J., Salamanca, J., Quiroz, R., Hernández, A., Hernández-Rojas, A., y Quesada, B.(2012). Identificación de factores de riesgo de embarazo en población adolescente escolar urbana y rural colombiana. Rev. Salud pública, 14(3), 404-416

González, J., y de la Hoz, F. (2011). Relaciones entre los comportamientos de riesgo psicosociales y la familia en adolescentes de Suba, Bogotá. Rev. Salud pública, 13(1),67-78

Grossman, J. M., Richer, A. M., Charmaraman, L., Ceder, I., y Erkut, S. (2018). Youth Perspectives on Sexuality Communication With Parents and Extended Family. Family Relations, 67(3), 368-380. http://dx.doi.org/10.1111/fare.12313

Hernández, L., Cargill, N., y Gutiérrez, G. (2012). Funcionalidad familiar y conducta de riesgo en estudiantes de nivel medio superior Jonuta, Tabasco 2011. Salud en Tabasco, 18(1),14-24. Disponible en: https://www.redalyc.org/articulo.oa?id=487/48724427004

Instituto Nacional de Estadísticas y Censos. (2014). Encuesta Nacional de Salud, Salud Reproductiva y Nutrición (ENSANUT). Presentación de principales resultados ensalud sexual y reproductiva. Disponible en: http://www.ecuadorencifras.gob.ec/category/ensanut/ 
Kalina, O., Geckova, A. M., Klein, D., Jarcuska, P., Orosova, O., van Dijk, J. P., y Reijneveld, S. A. (2011). Psychosocial factors associated with sexual behaviour in early adolescence. The European Journal of Contraception \& Reproductive Health Care, 16(4), 298-306. http://dx.doi.org/10.3109/13625187.2011.586076

Kalina, O., Geckova, A. M., Klein, D., Jarcuska, P., Orosova, O., van Dijk, J. P., y Reijneveld, S. A. (2013). Mother's and father's monitoring is more important than parental social support regarding sexual risk behaviour among 15-year-old adolescents. The European Journal of Contraception \& Reproductive Health Care, 18(2), 95-103. http://dx.doi.org/10.3109/13625187.2012.752450

Lavielle, P., Jiménez, F., Vázquez, A., Aguirre, C., Castillo, M., y Vega, S. (2014). Impactode la familia en las conductas sexuales de riesgo de los adolescentes. Rev. Med. Inst.Mex Seguro Soc, 52(1), 38-43

Lee, Y. M., Cintron, A., y Kocher, S. (2014). Factors Related to Risky Sexual Behaviors andEffective STI/HIV and Pregnancy Intervention Programs for African American Adolescents. Public Health Nursing, 31(5), 414-427. http://dx.doi.org/10.1111/phn.12128

Lee, Y.-M., Dancy, B., Florez, E., y Holm, K. (2013). Factors Related to Sexual Practices andSuccessful Sexually Transmitted Infection/HIV Intervention Programs for Latino Adolescents. Public Health Nursing, 30(5), 390-401. http://dx.doi.org/10.1111/phn.12039

Lee, Y.-M., Florez, E., Tariman, J., McCarter, S., y Riesche, L. (2015). Factors related tosexual behaviors and sexual education programs for Asian-American adolescents.

Applied Nursing Research, 28(3), 222-228. http://dx.doi.org/10.1016/j.apnr.2015.04.015

Lefkowitz, y Gillen. (2010). Adultez emergente y temprana. En D. Papalia, S. Wendkos, y R.Duskin, Desarrollo Humano (pág. 431). Mc Graw Hill.

Malcolm, S., Huang, S., Cordova, D., Freitas, D., Arzon, M., Jimenez, G. L., ... Prado, G.(2012). Predicting Condom Use Attitudes, Norms, and Control Beliefs in HispanicProblem Behavior Youth. Health Education \& Behavior, 40(4), 384-391. http://dx.doi.org/10.1177/1090198112440010

Maness, S., y Buhi, E. (2016). Associations Between Social Determinants of Health andPregnancy Among Young People: A Systematic Review of Research Published During the Past 25 Years. Public Health Reports, 131, 86-98

McCubbin, H. I., Thompson, A. I., y McCubbin, M. A. (1996). Family assessment: resiliency, coping and adaptation. Inventories for research and practice. Madison, Winsconsin: University of Winsconsin Publishers.

Mmari, K., Kalamar, A. M., Brahmbhatt, H., y Venables, E. (2016). The Influence of theFamily on Adolescent Sexual Experience: A Comparison between Baltimore andJohannesburg. PLOS ONE, 11(11), e0166032. http://dx.doi.org/10.1371/journal.pone.0166032

Mmbaga, E. J., Leonard, F., y Leyna, G. H. (2012). Incidence and Predictors of Adolescent'sEarly Sexual Debut after Three Decades of HIV Interventions in Tanzania: A Time toDebut Analysis. PLoS ONE, 7(7), e41700. http://dx.doi.org/10.1371/journal.pone.0041700

Moral de la Rubia, J., y Garza, D. (2017). Relación entre los estilos parentales y las conductassexuales de 
riesgo en adolescentes escolarizados de Monterrey, Nuevo León. Revista Perspectivas Sociales, 19(1), 41-65

Murry, V. M., Berkel, C., Chen, Y., Brody, G. H., Gibbons, F. X., y Gerrard, M. (2011).

Intervention Induced Changes on Parenting Practices, Youth Self-Pride and SexualNorms to Reduce HIVRelated Behaviors Among Rural African American Youths.Journal of Youth and Adolescence, 40(9), 1147-1163. http://dx.doi.org/10.1007/s10964-011-9642-x

Murry, V. M., Simons, R. L., Simons, L. G., y Gibbons, F. X. (2013). Contributions of familyenvironment and parenting processes to sexual risk and substance use of rural AfricanAmerican males: A 4-year longitudinal analysis. American Journal of Orthopsychiatry, 83(2-3), 299-309. http://dx.doi.org/10.1111/ajop.12035

Okigbo, C. C., Kabiru, C. W., Mumah, J. N., Mojola, S. A., y Beguy, D. (2015). Influence ofparental factors on adolescents' transition to first sexual intercourse in Nairobi, Kenya: a longitudinal study. Reproductive Health, 12(1). http://dx.doi.org/10.1186/s12978-015-0069-9

Olson, D., Sprenkle, D. y Russell, C. (1979). Circumplex model of marital and familysystems I: Cohesion and adaptability dimensions, family types and clinical applications. Family Process, 18, 3-28.

Olson, D.H., Portner, J. y Lavee, Y. (1985). Manual de la Escala de Cohesión y Adaptabilidad Familiar (FACES III Manual). Minneapolis: Life Innovation

Orcasita, L., Uribe, A., Castellanos, L., y Gutiérrez, M. (2012). Apoyo social y conductassexuales de riesgo en adolescentes del municipio de Lebrija-Santander. Revista dePsicología, 30(2), 371-406

Organización Mundial de la Salud. (2018). Organización Mundial de la Salud. El embarazo en la adolescencia. Disponible en: https://www.who.int/maternal_child_adolescent/topics/adolescence/dev/es/

Orihuela, C., Mrug, S., Davies, S., Elliott, M., Tortolero, S., Peskin, M... Schuster, M. (2020). Neighborhood Disorder, Family Functioning, and Risky Sexual Behaviors in Adolescence. J Youth Adolescence 49, 991-1004. https://doi.org/10.1007/s10964-020-01211-3

Papalia, D., Wendkos, S., y Duskin, R. (2010). Desarrollo Humano. Mc Graw Hill.

Pérez, R., Agurto, K., Contreras, K., Medina, L., Muñoz, C., Parra, J., y Sáez, K. (2012).Vulnerabilidad social y conductas sexuales de riesgo en un grupo de adolescenteschilenos, 2009. Estudio de corte transversal. Revista Colombiana de Obstetricia y Ginecología, 63(4). 327-333

Rhucharoenpornpanich, O., Chamratrithirong, A., Fongkaew, W., Miller, B. A., Cupp, P. K.,Rosati, M. J., ... Chookhare, W. (2012). Parent-Teen Communication about Sex in

Urban Thai Families. Journal of Health Communication, 17(4), 380-396. http://dx.doi.org/10.1080/10810730.2011.626668

Ryan, J., Roman, N., y Okwany, A. (2015). The Effects of Parental Monitoring andCommunication on Adolescent Substance Use and Risky Sexual Activity: ASystematic Review. The Open Family Studies Journal, 7(1) 12-27

Sámano, R., Martínez-Rojano, H., Robichaux, D., Rodríguez-Ventura, A. L., Sánchez- Jiménez, B., de la Luz Hoyuela, M., ... Segovia, S. (2017). Family context and individual situation of teens before, during 
and after pregnancy in Mexico City. BMC Pregnancy and Childbirth, 17(1). http://dx.doi.org/10.1186/s12884-017-1570-7

Udell, W., Donenberg, G., y Emerson, E. (2011). Parents matter in HIV-risk among probationyouth. Journal of Family Psychology, 25(5), 785-789. http://dx.doi.org/10.1037/a0024987

Van de Bongardt, D., de Graaf, H., Reitz, E., y Deković, M. (2014). Parents as Moderators ofLongitudinal Associations Between Sexual Peer Norms and Dutch Adolescents' Sexual Initiation and Intention. Journal of Adolescent Health, 55(3), 388-393. http://dx.doi.org/10.1016/j.jadohealth.2014.02.017

Voisin, D. R., Tan, K., Tack, A. C., Wade, D., y DiClemente, R. (2012). Examining ParentalMonitoring as a Pathway From Community Violence Exposure to Drug Use, Risky Sex, and Recidivism Among Detained Youth. Journal of Social Service Research, 38(5), 699-711. http://dx.doi.org/10.1080/01488376.2012.716020

Whittemore, R., y Knafl, K. (2005). The integrative review: Updated methodology. Journalof Advanced Nursing, 52(5), 546-55

Yi, S., Poudel, K. C., Yasuoka, J., Palmer, P. H., Yi, S., y Jimba, M. (2010). Role of risk andprotective factors in risky sexual behavior among high school students in Cambodia. BMC Public Health, 10(1). http://dx.doi.org/10.1186/1471-2458-10-477

Zambrano, G., Vera, S., y Flórez, L. (2012). Relación entre funcionalidad familiar y las estrategias de afrontamiento utilizadas por adolescentes embarazada. Revista Cienciay Cuidado, 9(2), 9-16 